\title{
Reliability Model for MEMS Accelerometers
}

\author{
Xingguo Xiong ${ }^{1}$, Yu-Liang $\mathrm{Wu}^{2}$, Wen-Ben Jone ${ }^{3}$, \\ 1 Department of Electrical and Computer Engineering, University of Bridgeport, Bridgeport, CT 06604, USA \\ 2 Dept. of Computer Science \& Engineering, The Chinese University of Hong Kong, Shattin, Hong Kong \\ 3 Department of ECECS, University of Cincinnati, Cincinnati, OH 45221, USA \\ Email: 1 xxiong@bridgeport.edu, 2 ylw@cse.cuhk.edu.hk, ${ }^{3}$ wjone@ececs.uc.edu
}

\begin{abstract}
MEMS (Microelectromechanical System) reliability is a very critical issue for its commercial applications. In order to measure the reliability of MEMS, a systematic reliability model is required. In this paper, we developed a MEMS reliability model for quantitative assessment of the MEMS reliability analysis. Based on this model, we analyze the reliability of both BISR (built-in-self-repairable) and nonBISR MEMS comb accelerometers under Z-axis shocking environment. Simulation results demonstrate very effective reliability enhancement due to the BISR design. The reliability model can also be applied to other MEMS devices under various failure mechanisms in a similar way.
\end{abstract}

Keywords: MEMS (Microelectromechanical System), redundancy repair, reliability, accelerometer, fracture probability.

\section{INTRODUCTION}

In order for MEMS technologies to be used for real applications, yield as well as reliability are two very important issues which need to be immediately addressed. Reliability analysis is required for almost every commercial product. With the commercialization of MEMS devices, their reliabilities need to be thoroughly studied. Second, MEMS will be integrated into System-on-Chip (SoC) design very soon. The reliability of an entire SoC cannot be guaranteed if the reliability of MEMS is low. Further, MEMS is finding more and more applications in safetycritical areas, such as aerospace, medical instruments. For these applications, extremely high reliability is required. For example, during the launching process of a rocket, the failure of a tiny MEMS device may easily lead to unpredictable disaster. An unreliable bioMEMS chip embedded inside human body can be a serious threat to the health and may lead to the loss of life. Thus, the reliability research for MEMS in safety-critical applications is an especially urgent need.

Efforts on MEMS failure mechanisms and reliability research have been reported [1]-[6]. In [1], a comprehensive analysis on various MEMS defect sources, failure mechanisms and how they affect the MEMS reliability have been proposed. In [2][3], the MEMS material fatigue and aging under long-term cyclic loading has been discussed. In [4][5], MEMS reliability under shock and vibration environments has been explored. In [6], efforts have been made to explore the physical mechanism of stiction in surface micromachining and its impact on MEMS reliability. Although its importance has been widely recognized by researchers, MEMS reliability research still remains in its infant stage. Multiple energy domains are generally involved in the working principle of MEMS devices. Moreover, most MEMS devices contain movable components. Hence, MEMS devices are vulnerable to much more defect sources during its fabrication process and infield usage compared to VLSI chips. This makes MEMS reliability research a challenging work. The understanding of various MEMS failure mechanisms is also non-trivial. Many MEMS failure mechanisms are unclear yet. The interaction among different MEMS failure mechanisms and its influence on MEMS reliability are not well discussed. Furthermore, most of the MEMS reliability analysis methods are still qualitative. A well-developed quantitative MEMS reliability model is not available. In this work, we develop a MEMS reliability model which can be used to quantitatively evaluate the reliability of MEMS devices. The reliability model has been applied to quantitatively assess the reliability of our previously proposed self-repairable MEMS accelerometer [7]. Based on this model, we evaluate the reliabilities of both BISR (built-in self-repair) and nonBISR MEMS accelerometers under shock environments. A comparison of the simulation results demonstrates that the BISR design leads to an effective reliability enhancement when compared to the non-BISR device. Although here we analyze the MEMS reliability under shock environments as an example, the reliability model and the research strategies can also be applied to other MEMS devices under other failure mechanisms (e.g., material fatigue, stiction) in a similar way.

\section{RELIABILITY ANALYSIS OF NON-BIST/BISR MEMS ACCELEROMETERS}

MEMS comb accelerometer device contains a large number of finger groups which are necessary to ensure enough signal strength. However, the large number of finger groups unavoidably leads to the decrease in yield as well as reliability. In [7], we proposed a built-in self-repairable MEMS comb accelerometer device. The device consists of 
six identical modules (for example), and each module has its own beams, mass and finger structures (fixed and movable). By assumption, four modules are connected together as the main device, while the remaining two modules serve as redundancy. The movable parts of each module are physically connected to those of adjacent modules through the common anchors, and signals sensed by all movable fingers in the device are connected to the sensing circuit directly. However, the fixed fingers of each module are connected to the modulation signal circuit through switches made of analog MUXes. By turning on or off these switches, we can determine whether a module works as part of the main device or the redundant device. If a module is tested as faulty, the control circuit will permanently exclude the module from the main device and replace it with a good redundant module (if there is any). Thus, after repairing, the main device can still be ensured to work properly. Because each module has its own independent beam and mass structure, a faulty module does not affect the function of other modules. For example, even if the movable part of one module is broken or stuck to substrate, the movable parts of other modules can still move freely and work jointly to ensure the function of the main device. Our previous analysis in [7] demonstrates that the BISR design leads to effective yield increase. In this paper, we will develop a reliability model and evaluate the effectiveness of reliability enhancement due to the BISR design. In the following discussion, we call the MEMS comb accelerometer with (without) the BISR feature as a BISR (non-BISR) accelerometer. In order for a fair comparison, we assume that the total number of finger groups of the non-BISR accelerometer should equal to that of the main device in the BISR accelerometer.

\section{A. Basic Concepts of Reliability}

Reliability is the probability that a component, equipment, or system will perform the required function under different operating conditions encountered for a stated period of time [8]. The reliability function is denoted by $R(t)(0 \leq R(t) \leq 1)$ where $t$ is time. The larger the reliability function value is, the more reliable the component, equipment or system will be. Meanwhile, the unreliability $Q(t)$, or the probability of failure, is defined as the probability that a component, equipment, or system will not perform the required function under the operating conditions encountered for a stated period of time $t$. Apparently, we have $R(t)+Q(t)=1$. The failure rate $\lambda$ is expressed as the ratio of the total number of failures to the total operating time: $\lambda=K / T$, where $K$ is the number of failures and $T$ is the total operating time. For most products, $\lambda$ is generally a very small number. Its unit can be number of failures per $1 \times 10^{6}$ hours. As we can see, the larger the $\lambda$ value is, the more unreliable the component, equipment or system will be.
The Mean-Time-To-Failures (MTTF) is the reciprocal of the failure rate: $M T T F=1 / \lambda$.

In reality, the failure rate $\lambda$ is generally the function of time. The failure rate $\lambda(t)$ of mechanical components, VLSI chips and MEMS devices follows the behavior of a bathtub curve as shown in Figure 1 [8]. It is believed the failure rate of MEMS devices also follows the bathtub curve. The bathtub function consists of three regions. In the initial stage, the failure rate is high due to the latent defects in the device, and falls off till time $t_{\text {infat }}$. After that, the device enters a stable stage with a constant failure rate. For high reliability applications, this constant failure rate $\lambda$ should be extremely small. Finally, after time $t_{\text {operation, }}$ the failure rate will increase sharply due to wear-out and the device comes to the end of its lifetime. The useful time of the device with low constant failure rate is defined as $t_{\text {useful }}=t_{\text {operation }}-t_{\text {infant }}$. Generally, $t_{\text {infatand }}$ and $t_{\text {operation }}$ of a certain product cannot be given as definite values. The manufacturers will give average values for their products based on statistical testing.

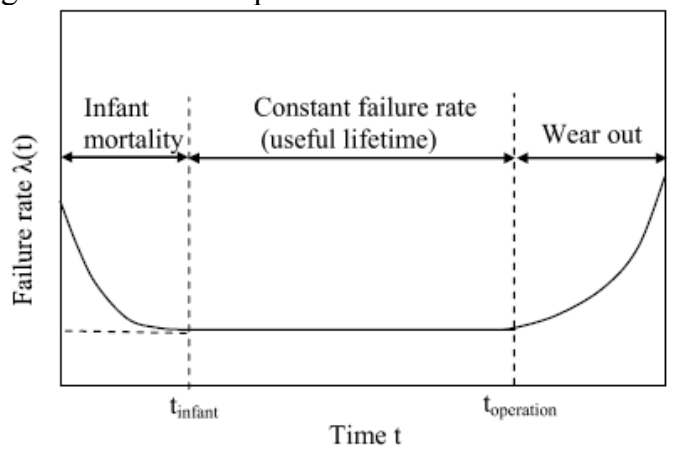

Figure 1. The bathtub curve of failure rate [8]

There are several standard probability models available for describing the reliability of a system: the exponential reliability distribution, the binomial reliability distribution, the Poisson reliability distribution, and Weibull reliability distribution [8].

1) The exponential reliability distribution

The exponential reliability distribution is the most common probability model used to predict the lifetime of a system. Thus, in our MEMS reliability analysis, the exponential reliability model is used. The reliability function $R(t)$ is expressed as

$R(t)=e^{-\lambda t}$

where $\lambda$ is the failure rate and $t$ is the time period. The unreliability $Q(t)$ is therefore

$Q(t)=1-R(t)=1-e^{-\lambda t}$

The exponential reliability distribution is shown in Figure 2. 


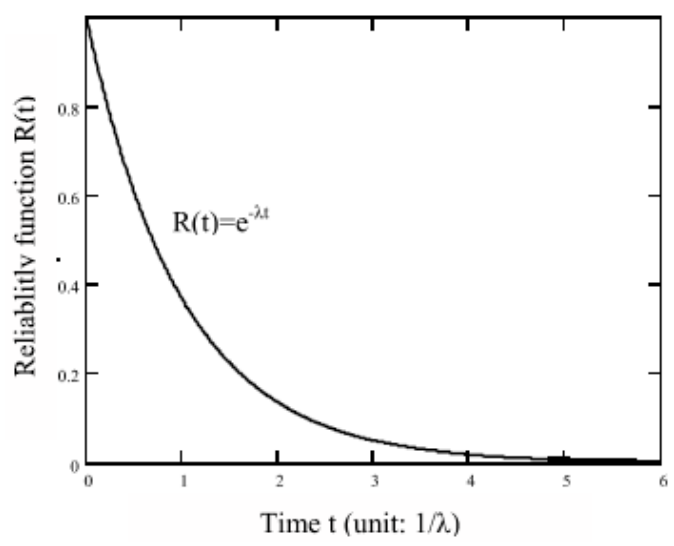

Figure 2. The exponential reliability distribution function.

2) The binomial reliability distribution

The binomial reliability distribution is used for describing the reliability of a discrete distribution. It can be expressed as $(R+Q)^{n}=1$, where $n$ is the total number of trials conducted. A typical example of binominal reliability distribution is the case of flipping coins for heads and tails. If we define the probability of obtaining a head and a tail as $\mathrm{R}$ and $\mathrm{Q}$ separately, we have $R=Q=0.5$. If two trials are made $(n=2)$, then $(R+Q)^{2}=R^{2}+2 R Q+Q^{2}=1$.

3) The Poisson reliability distribution

The Poisson reliability distribution is a discrete distribution which provides a useful tool in the case of the binomial distribution. The reliability can be expressed as

$R=1-\left(Q_{1}+Q_{2}+Q_{3}+\ldots\right)$

where $Q_{i}$ is the probability of exactly $i$ failures occurring during time period $\mathrm{t}$, and can be expressed as

$Q_{i}(t)=(\lambda t)^{i} e^{-\lambda t} / i$ !

The probability of zero failure in the Poisson reliability model comes to the result of the exponential reliability distribution $R_{0}(t)=e^{-\lambda t}$. That is, the exponential reliability distribution can be treated as a special case of Poisson reliability model for $i=0$.

4) The Weibull reliability distribution

The Weibull reliability function is expressed as

$R(T 1)=\exp \left\{-[(T 1-\gamma) / \eta]^{\beta}\right\}$

For general reliability measurement, we consider $\gamma=0$ and $\alpha=1 / \eta$, hence

$R(t)=\exp \left[-(\alpha t)^{\beta}\right]$

where $\beta$ is the shape parameter which indicates whether the failure rate is increasing or decreasing. If $\beta<1.0$, the failure rate is decreasing. If $\beta=1.0$, the failure rate is constant. If $\beta>1.0$, the failure rate is increasing. If $\beta=1.0$, it comes to the result of exponential distribution: $R(t)=e^{-(\alpha t)}$. Thus, the exponential distribution can also be treated as a special case of the Weibull reliability function for $\beta=1.0$.

The above reliability models describe the reliability of an individual component. However, sometimes we also need to evaluate the reliability of a system consisting of multiple components. Such system reliability models enable us to calculate the reliability characteristics of a design, evaluate design alternatives, and perform sensitivity analysis. Depending on the configuration of the system, different system reliability models (such as series, parallel, and k-outof-n, etc.) are available [8].

\section{1) Series Reliability Model}

If the functional operation of a system depends on the successful operation of all system components, the reliability of the system can be calculated with a series reliability model. The reliability block diagram of the series model is shown in Figure 3. Assume the system consists of nnumber of serial components, and the failure rate of each component $i$ is $\lambda_{i}$, the reliability of the entire series system is $R_{\text {tot }}=\prod_{i=1}^{n} R_{i}(t)=e^{-\lambda_{1} t} \cdot e^{-\lambda_{2} t} \ldots \cdot e^{-\lambda_{n} t}=e^{-\left(\sum_{i=1}^{n} \lambda_{i}\right) t}$

From the above equation, we have

$$
\lambda_{\text {tot }}=\sum_{i=1}^{n} \lambda_{i}
$$

That is, the failure rate of a series system is the sum of the failure rates of all the series components. The mean-time-tofailure (MTTF) of the system can be expressed as

$$
M_{T T F}=\sum_{i=1}^{n} \frac{1}{M T T F_{i}}
$$

where $\mathrm{MTTF}_{\text {tot }}$ is the MTTF of the entire series system, MTTF $_{i}$ is the MTTF of the $i^{\text {th }}$ component.

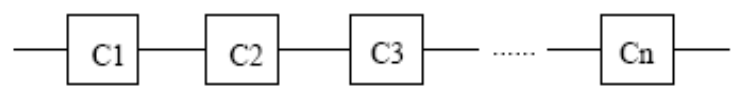

Figure 3. The block diagram of series reliability model 2) Parallel Reliability Model

The block diagram of a parallel reliability model is shown in Figure 4. The system functions properly until all of the components $\left(C_{1}\right.$ to $\left.C_{n}\right)$ fail. Hence, the reliability $R_{\text {tot }}$ of the parallel system is given as one minus the probability of failure for each component as shown below

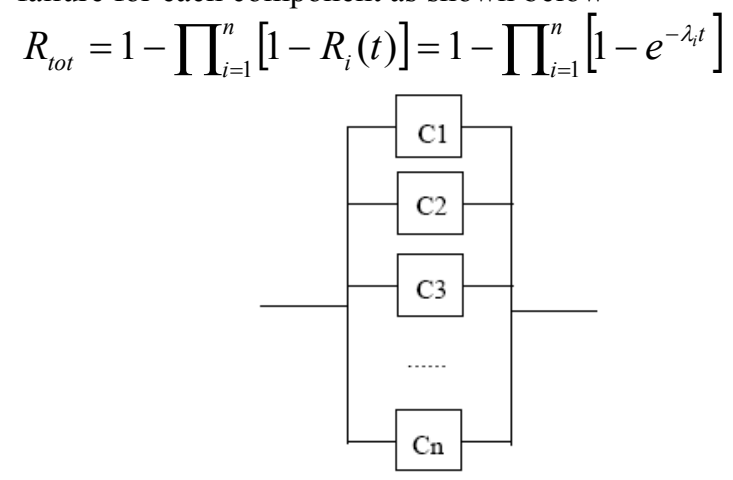

Figure 4. The block diagram of parallel reliability model.

3) k-out-of-n Redundancy Reliability Model 
The block diagram of a k-out-of-n redundancy reliability model is shown in Figure 5. Among the $n$ number of modulus, at least $k$ number of modules need to be fault-free in order for the whole system to work properly. In other words, a maximum of $\mathrm{n}-\mathrm{k}$ number of faulty modules are allowed without losing the function of the whole system. Our BISR comb accelerometer [7] is exactly an example of k-out-of-n reliability model. If the BISR accelerometer contains $n$ modules in the main device and $m$ redundant modules, it is a n-out-of- $(\mathrm{n}+\mathrm{m})$ model. Assume the reliability of each component as $R_{c}$. The reliability $R_{t o t}$ of the k-out-of-n redundancy system is given as below [8]

$R_{t o t}(t)=R_{c}^{n}(t)+n R_{c}^{n-1}(t)\left(Q_{c}(t)\right)+\ldots+\left(\begin{array}{l}n \\ k\end{array}\right)\left(R_{c}(t)\right)^{k}\left(Q_{c}(t)\right)^{n-k}$

Assume the failure rate of each component as $\lambda$, the MTTF of the system can be calculated as [8]

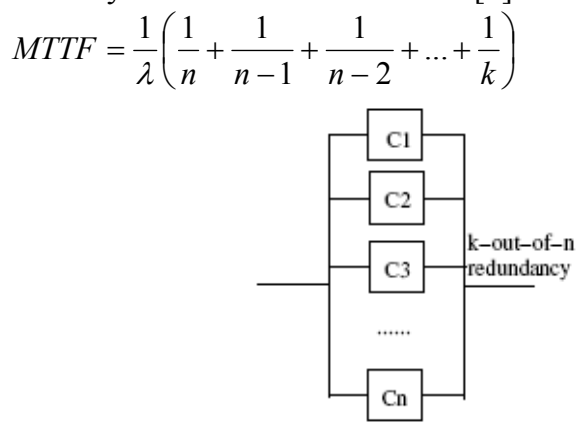

Figure 5. The block diagram of k-out-of-n redundancy reliability model.

\section{B. Reliability Model of Non-BISR MEMS Device}

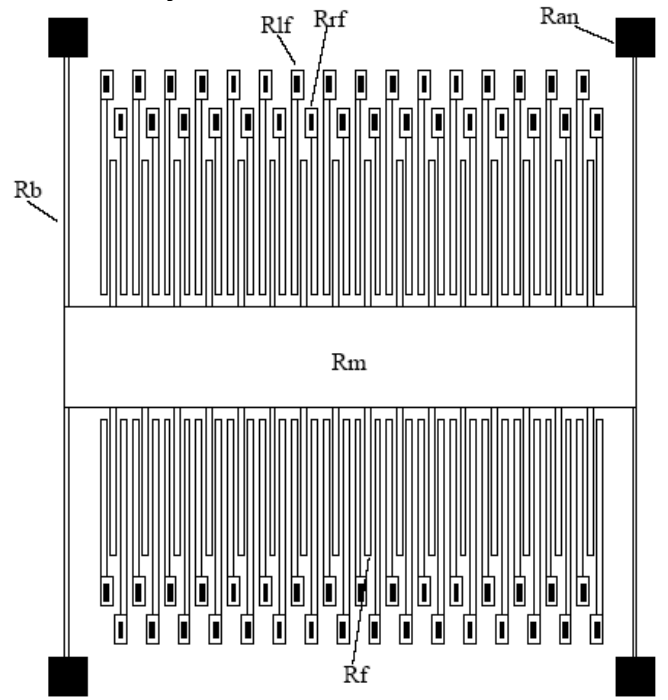

Figure 6. The structural diagram of a non-BISR MEMS device.

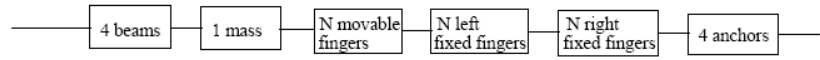

Figure 7. The reliability model for the non-BISR MEMS device.
The structural diagram of the non-BISR MEMS accelerometer [7][9] for this analysis is shown in Figure 6, and its corresponding reliability model is shown in Figure 7. As shown in Figure 6, the non-BISR device consists of four beams, one mass, $N$ number of movable fingers, $2 N$ number of left/right fixed fingers, and four anchors. All these components must be fault-free in order for the entire device to function correctly. If any of these components becomes faulty, the whole device will malfunction. For example, if one of the beams is broken, a movable finger is stuck to the corresponding fixed finger, or the mass is stuck to the substrate, then the device will not be able to work properly. Although the fixed components such as anchors and fixed fingers are less likely to be faulty than the movable components, they also must be fault-free in order to ensure the proper function of the device. For example, if one left fixed finger is shorted to its neighborhood right fixed finger, this will lead to a short circuit to the signal detection circuit. Or, if an anchor is lifted off the substrate, the device structure will be unstable. Thus, the reliability of a nonBISR device can be described as a series model, as shown in Figure 7. Since the four beams have exactly the same dimension and equal loading, we assume all four beams have the same reliability $R_{b}$. Similarly, we assume the reliabilities of the mass and each movable finger as $R_{m}$ and $R_{f}$ separately. The reliabilities of one fixed finger and one anchor are denoted as $R_{f f}$ and $R_{a n}$ individually. Finally, the reliability of the entire non-BISR device can be expressed as

$$
R_{n s r}=R_{b}^{4} \cdot R_{m} \cdot R_{f}^{N} \cdot R_{f f}^{2 N} \cdot R_{a n}
$$

In reality, movable components of the MEMS device are vulnerable to more defect sources when compared with fixed components. Thus, the reliabilities of fixed components can be much higher than those of movable components. The defects of fixed components mainly result from device fabrication. The devices with catastrophic defects in fixed components can be filtered out during manufacturing test. During in-field usage, it is less likely for the fixed components to develop new defects compared to the movable components. Thus, in the following discussion, we will mainly concentrate on the reliability of the movable components, and the reliability of the fixed parts can be treated as constant 1 . In this way, the reliability of the nonBISR device can be expressed as

$$
R_{n s r}=R_{b}^{4} \cdot R_{m} \cdot R_{f}^{N}
$$

From the reliability model of the non-BISR device, we can see that the major threat to the device reliability comes from the large index (i.e., $N$ ) of $R_{f}$. For example, if the reliability of a single movable finger is 0.99 and $N$ equals 42 , this will reduce the entire device reliability to 0.656 even though the beam and mass are assumed perfect. This is the major impetus for us to implement the redundancy repair technique for MEMS comb accelerometers. By 
modularizing the device, each module contains a smaller number of comb fingers. The reliability of each module will be higher than the original non-BISR device. By implementing the redundancy repair technique, even higher reliability can be achieved for the entire device. Assume the failure rates for the beam, mass and finger as $\lambda_{b}, \lambda_{m}$ and $\lambda_{f}$ separately. The reliability functions of the beam, mass and finger can be expressed as

$R_{b}=e^{-\lambda_{b} t}, R_{m}=e^{-\lambda_{m} t}, R_{f}=e^{-\lambda_{f} t}$.

Hence, the reliability function of the non-BISR device with respect to time $t$ is

$R_{n s r}(t)=e^{-\lambda_{n s r} t}=e^{-\left(4 \lambda_{b}+\lambda_{m}+N \lambda_{f}\right) t}$

where the failure rate $\lambda_{n s r}$ of the non-BISR device is

$\lambda_{n s r}=4 \lambda_{b}+\lambda_{m}+N \lambda_{f}$

Once we know the failure rates $\lambda_{b}, \lambda_{m}$ and $\lambda_{f}$, we can derive the reliability of the non-BISR device at certain time $t$ based on the equation for $R_{n s r}(t)$.

\section{Reliability Model of BISR MEMS Device}

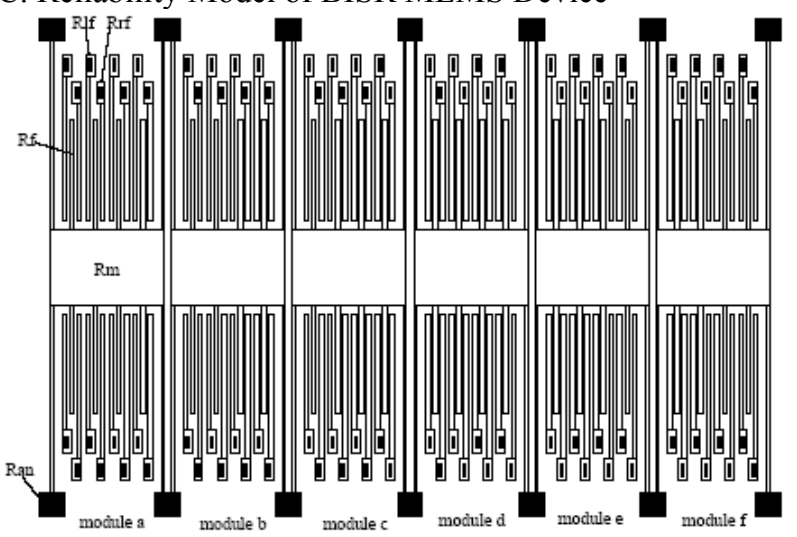

Figure 8. The structural diagram of the BISR MEMS accelerometer.

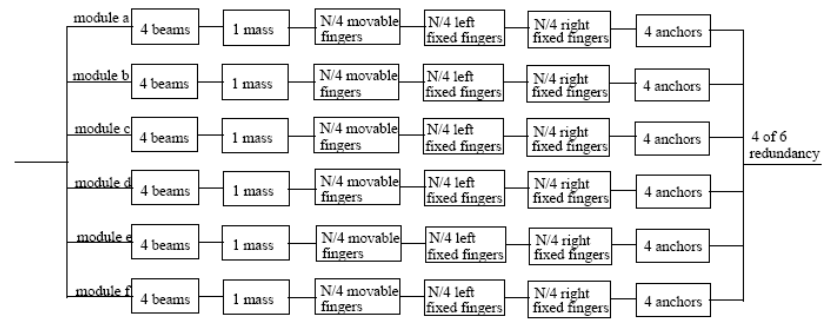

Figure 9. Reliability model for BISR device.

The structural diagram of the BISR comb accelerometer [7] is shown in Figure 8 and its corresponding reliability model is shown in Figure 9. The BISR comb accelerometer consists of six identical modules. In each module, it consists of four beams, one smaller mass, $(N / 4)$ number of movable fingers, $(N / 2)$ number of left and right fixed fingers, and four anchors. Note that $N$ is the number of movable fingers for the non-BISR device. Again, the reliability of each module can be described with a series model. Assume the reliabilities of each beam, movable finger and mass as $R_{b r}$, $R_{f r}$ and $R_{m r}$ separately, and the reliabilities of each fixed finger and anchor as $R_{f f}$ and $R_{a n}$ individually. The reliability $R_{\text {mod }}$ of each BISR module can be expressed as

$$
R_{\text {mod }}=R_{b r}^{4} \cdot R_{m r} \cdot R_{f}^{N / 4} \cdot R_{f f}^{N / 2} \cdot R_{a n}
$$

Similarly, if we only concentrate on the reliability of movable components, the reliability of each module can be expressed as

$$
R_{\text {mod }}=R_{b r}^{4} \cdot R_{m r} \cdot R_{f}^{N / 4}
$$

Assume the failure rates for the beam, mass and finger of each BISR module as $\lambda_{b r}, \lambda_{m r}$ and $\lambda_{f}$ separately. The reliability functions of the beam, mass and finger can be expressed as

$$
R_{b r}=e^{-\lambda_{b r} t}, R_{m}=e^{-\lambda_{m r} t}, R_{f}=e^{-\lambda_{f r} t}
$$

Hence, the reliability function of the entire BISR module is

$$
R_{\text {mod }}(t)=e^{-\left(\lambda_{\text {mod }} t\right)}=e^{-\left[4 \lambda_{b r}+\lambda_{m r}+(N / 4) \lambda_{f}\right] t}
$$

where the failure rate $\lambda_{\text {mod }}$ of each module is

$$
\lambda_{\text {mod }}=4 \lambda_{b r}+\lambda_{m r}+(N / 4) \lambda_{f}
$$

The unreliability $Q_{\text {mod }}$ of each module is

$$
Q_{\text {mod }}(t)=1-R_{\text {mod }}(t)
$$

There are totally six identical modules in the BISR comb accelerometer. Among them, only four modules are required to be fault-free to ensure the proper function of the device. The reliability of such a system can be calculated using a kout-of-n redundancy reliability model. The reliability $R_{s r}$ of the BISR accelerometer can be expressed as

$$
\begin{aligned}
& R_{s r}(t)=R_{\text {mod }}^{6}(t)+6 R_{\text {mod }}^{5}(t) Q_{\text {mod }}(t)+15 R_{\bmod }^{4}(t) Q_{\text {mod }}^{2}(t) \\
& =\left(e^{-\lambda_{\bmod }(t)}\right)^{6}+6\left(e^{-\lambda_{\bmod }(t)}\right)^{5}\left(1-e^{-\lambda_{\bmod }(t)}\right)+15\left(e^{-\lambda_{\bmod }(t)}\right)^{4}\left(1-e^{-\lambda_{\bmod }(t)}\right)^{2}
\end{aligned}
$$

This is the reliability function of a 4-out-of-6 redundancy BISR comb accelerometer. For the general case, assume there are $n$ number of modules in the main device and $m$ number of modules as redundancy. The reliability of the BISR accelerometer can be expressed as

$$
\begin{aligned}
& R_{s r}(t)=\left(R_{\text {mod }}(t)\right)^{n+m}+(n+m)\left(R_{\text {mod }}(t)\right)^{n+m-1}\left(Q_{\text {mod }}(t)\right)+\ldots \\
& +\left(\begin{array}{c}
n+m \\
n
\end{array}\right)\left(R_{\text {mod }}(t)\right)^{n}\left(Q_{\text {mod }}(t)\right)^{m}
\end{aligned}
$$

\section{Reliability Enhancement and Reliability Analysis}

After we have developed the reliability models for both non-BISR and BISR MEMS devices, we can derive the reliability increase $I R(t)$ due to redundancy repair by

$$
\operatorname{IR}(t)=R_{s r}(t)-R_{n s r}(t)
$$

In the BISR MEMS comb accelerometer, the large number of comb finger groups are divided into several modules. Each module contains a smaller number of comb finger groups, and thus the risk for each module to be faulty is lowered. The redundancy further improves the reliability of the BISR device. However, compared to the original nonBISR device, the BISR device contains more beams. This will lead to the decrease of the reliability. The net reliability 
increase or decrease depends on the interaction between these counteracting factors.

\section{Reliability Analysis Result}

Based on the above reliability model, we can simulate the reliability increase due to redundancy repair for BISR MEMS accelerometers compared to non-BISR design. For example, we evaluated the reliabilities of example designs for both non-BISR and BISR devices under shock environment, and assume fracture as the major failure mechanism. The non-BISR device has a beam width of $W_{b n s r}=3.2 \mu \mathrm{m}$. For BISR device, we consider two cases [10]: (1). BWC device: the BISR device with Beam Width Compensation (i.e., shrinking the beam width to compensate the sensitivity loss due to BISR design) with

$W_{b b w c}=2.0 \mu m=0.63 W_{b n s r}$.

(2). EFC device: the BISR device with Electrostatic Force Compensation (i.e., using electrostatic force to compensate the sensitivity loss) with $W_{b e f c}=3.2 \mu m=W_{b n s r}$.

We used ANSYS simulation to extract the maximum stress $\sigma_{\max }$ values corresponding to about 30 different $Z$-axis shock accelerations from $0 g$ to $10^{5} g$. Based on these data, we can calculate the Weibull fracture probability $\left(P_{f}\right)$ [11] and the shock reliability $\left(R_{f}\right)$ values for non-BISR and BWC/EFC BISR devices corresponding to each individual shock acceleration. The plots of the simulated Z-axis shock reliability versus each shock acceleration of nonBISR/BISR devices are shown in Figure 10. As shown in the Figure 10, the shock reliability of the EFC BISR device is larger than that of the BWC BISR device, and both are larger than the non-BISR device. We can see that if the Zshock acceleration falls into the range between the mean fracture stress of non-BISR device and BISR devices, a (maximum) reliability increase as large as 1 can be achieved due to the BISR design.

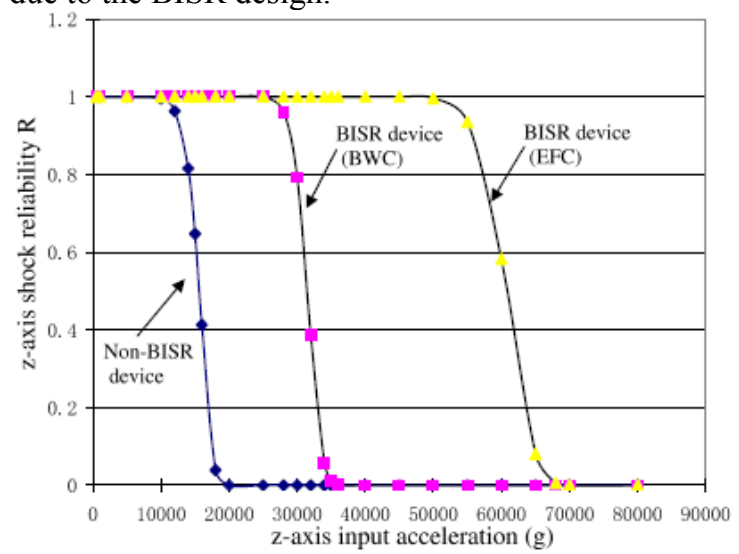

Figure 10. The z-axis shock reliability for non-BISR/BISR devices.

\section{CONCLUSIONS}

In this paper, a quantitative MEMS reliability model has been developed. Based on this reliability model, the reliabilities of both non-BISR and BISR MEMS comb accelerometer devices under shock environment have been assessed. The Weibull fracture probability function has been used to evaluate the fracture probability of MEMS accelerometers under Z-axis shock acceleration. The simulation results demonstrate an effective reliability enhancement for both the BWC and EFC BISR comb accelerometers, compared to non-BISR design. In the future research, we will also try to further improve our MEMS reliability model to account for other MEMS devices under various failure mechanisms. In this way, the reliability model can be used to assess and predict the MEMS reliability for various MEMS devices under various failure mechanisms. It can also guide us in improving the MEMS reliability for safety-critical applications.

\section{REFERENCES}

[1] B. Stark (editor), "MEMS Reliability Assurance Guidelines for Space Applications", Jet Propulsion Laboratory Publication 99-1, Pasadena, USA, Jan. 1999.

[2] M. Tabib-Azar, K. Wong, andW. Ko, "Aging Phenomena in heavily doped $(\mathrm{p}+)$ micromachined silicon cantilever beams", Sensors and Actuators A, Vol. 33, pp. 199-206, 1992.

[3] C. L. Muhlstein, R. T. Howe, and R. O. Ritchie, "Fatigue of polycrystalline silicon for microelectromechanical system applications: crack growth and stability under resonant loading conditions", Mechanics of Materials, Vol. 36, pp. 1333, 2004

[4] D. M. Tanner, J. A.Walraven, K. Helgesen, L.W. Irwin, F. Brown, N. F. Smith, and N. Masers, "MEMS reliability in shock environments", Proceedings of IEEE International Reliability Physics Symposium, San Jose, CA, USA, pp. 129138, Apr. 10-13, 2000.

[5] D. M. Tanner, J. A. Walraven, K.S.Helgesen, L. W. Irwin, D. L. Gregory, J. R. Stake, and N. F. Smith, "MEMS reliability in a vibration environment", IEEE 38th Annual International Reliability Physics Symposium, San Jose, California, USA, pp. 139-145, 2000.

[6] W. M. V. Spengen, R. Puers, and I. D. Wolf, "On the physics of stiction and its impact on the reliability of microstructures", Journal of Adhesion Science and Technology, Vol. 17, No. 4, pp. 563-582, 2003.

[7] X. Xiong, Y. Wu, and W. Jone, "Design and analysis of selfrepairable MEMS accelerometer," Proceedings of the 20th IEEE International Symposium on Defect and Fault Tolerance in VLSI Systems (DFT'05), Monterey, CA, USA, pp. 21-29, Oct. 3-5, 2005.

[8] K. Neubeck, Practical Reliability Analysis, Pearson Prentice Hall, New Jersey, USA, 2004.

[9] W. Kuehnel, and S. Sherman, "A Surface Micromachined Silicon Accelerometer with On-chip Detection Circuitry," Sensors and Actuators, Vol. A, Issue 45, pp. 7-16, 1994.

[10] X. Xiong, Built-in self-test and self-repair for capacitive MEMS devices, Ph.D dissertation, University of Cincinnati, July 2005.

[11] S. Greek, F. Ericson, S. Johansson, and J. Schweitz, "In situ tensile strength measurement and Weibull analysis of thick film and thin film micromachined polysilicon structure", Thin Solid Films, Vol. 292, pp. 247-254, 1997. 